\title{
Emotion
}

\section{Affect Dynamics in Relation to Depressive Symptoms: Variable, Unstable or Inert?}

Peter Koval, Madeline L. Pe, Kristof Meers, and Peter Kuppens

Online First Publication, August 5, 2013. doi: 10.1037/a0033579

CITATION

Koval, P., Pe, M. L., Meers, K., \& Kuppens, P. (2013, August 5). Affect Dynamics in Relation to Depressive Symptoms: Variable, Unstable or Inert?. Emotion. Advance online publication. doi: $10.1037 / a 0033579$ 


\title{
Affect Dynamics in Relation to Depressive Symptoms: Variable, Unstable or Inert?
}

\author{
Peter Koval, Madeline L. Pe, and Kristof Meers \\ KU Leuven - University of Leuven
}

\author{
Peter Kuppens \\ KU Leuven - University of Leuven and University of \\ Melbourne
}

\begin{abstract}
Depression not only involves disturbances in prevailing affect, but also in how affect fluctuates over time. Yet, precisely which patterns of affect dynamics are associated with depressive symptoms remains unclear; depression has been linked with increased affective variability and instability, but also with greater resistance to affective change (inertia). In this paper, we argue that these paradoxical findings stem from a number of neglected methodological/analytical factors, which we address using a novel paradigm and analytic approach. Participants $(N=99)$, preselected to represent a wide range of depressive symptoms, watched a series of emotional film clips and rated their affect at baseline and following each film clip. We also assessed participants' affect in daily life over 1 week using experience sampling. When controlling for overlap between different measures of affect dynamics, depressive symptoms were independently associated with higher inertia of negative affect in the lab, and with greater negative affect variability both in the lab and in daily life. In contrast, depressive symptoms were not independently related to higher affective instability either in daily life or in the lab.
\end{abstract}

Keywords: depression, emotion/affect dynamics, inertia, variability, instability

How a person feels, on the average, is telling of their level of well-being and psychological adjustment. Indeed, disturbances in prevailing affect are core symptoms of many mental disorders (e.g., Keltner \& Kring, 1998; Rottenberg \& Johnson, 2007). However, feelings are not invariant states, but rather dynamical processes that fluctuate in response to environmental demands and regulatory forces (Kuppens, Oravecz, \& Tuerlinckx, 2010). Thus, in addition to studying average levels of affect, examining patterns of affective fluctuations, or affect dynamics, may provide crucial insights into psychological functioning and well-being (Bylsma \& Rottenberg, 2011; Hollenstein, Lichtwarck-Aschoff, \& Potworowski, 2013; Wang, Hamaker, \& Bergeman, 2012).

Depression $^{1}$ is a case in point; while it is well established that depression is associated with higher average levels of negative affect (NA) and lower average levels of positive affect (PA; Watson, Clark, \& Carey, 1988), recent research indicates that depression also involves alterations in affect dynamics (Kuppens,

Peter Koval, Madeline L. Pe, and Kristof Meers, Department of Psychology and Educational Sciences, KU Leuven - University of Leuven, Leuven, Belgium; Peter Kuppens, Department of Psychology and Educational Sciences, KU Leuven - University of Leuven and Department of Psychological Sciences, University of Melbourne, Parkville, Australia.

Peter Koval is a Doctoral Research Fellow with the Research Foundation-Flanders (FWO). This research was supported by KU Leuven Research Council Grants OT/11/031 and GOA/10/02, and by a grant from the FWO. We thank Philippe Verduyn and Francis Tuerlinckx for their comments.

Correspondence concerning this article should be addressed to Peter Koval, KU Leuven, Tiensestraat 102, 3000 Leuven, Belgium. E-mail: peter.koval@ppw.kuleuven.be
Allen, \& Sheeber, 2010; Peeters, Berkhof, Delespaul, Rottenberg, \& Nicolson, 2006; Thompson et al., 2012). However, the literature is ambiguous regarding the specific patterns of affective fluctuations associated with depression. As we review below, some studies report increased affective variability and instability among depressed individuals (e.g., Thompson et al., 2012), while others link depression with greater resistance to affective change (e.g., Kuppens, Allen, et al., 2010), suggesting seemingly opposite patterns of affect dynamics.

Reconciling these paradoxical findings is not merely an intellectual pursuit. Rather, identifying the specific patterns of affect dynamics associated with depressive symptoms may shed light on the specific affective dysregulation involved in depression (Campbell-Sills \& Barlow, 2007). This may have implications for recognizing depression vulnerability among currently healthy individuals (Kuppens et al., 2012) as well as for the diagnosis and treatment of clinical depressive disorders. The current study aimed to address this important aim by pinpointing the specific patterns of affect dynamics associated with depressive symptoms using a novel paradigm and analytic approach.

\section{The Paradox of Affect Dynamics in Depression}

A number of studies have shown that depression is associated with higher affective variability, measured as the within-person variance or standard deviation $(S D)$ of affect over time (e.g., Peeters et al., 2006; Wichers et al., 2010). While this finding

\footnotetext{
${ }^{1}$ We use depression as a general term referring to both the clinical syndrome known as Major Depressive Disorder (MDD; American Psychiatric Association, 2000) and elevated depressive symptoms that may not meet diagnostic criteria for MDD. Where relevant, we distinguish between $M D D$ and depressive symptoms.
} 
indicates that depression is related to the experience of a large range of affect levels, it has been misinterpreted as evidence that depressed individuals are "more emotionally labile" (Silk, Steinberg, \& Morris, 2003, p. 1870). In fact, affective lability or instability implies not only high overall variability, but also frequent moment-to-moment fluctuations. Variability indices such as the $S D$ do not capture this temporal dependency component of instability (Ebner-Priemer, Eid, Kleindienst, Stabenow, \& Trull, 2009; Jahng, Wood, \& Trull, 2008). Thus, a person whose affect level increased linearly over time would have a high level of affective variability (e.g., high $S D$ ), but could hardly be labeled as affectively unstable (Larsen, 1987).

In view of this, Jahng et al. (2008) proposed the mean square successive difference (MSSD) as a true measure of affective instability that incorporates both variability and temporal dependency. A number of studies using MSSD or similar indices have linked depression with greater affective instability (e.g., Neumann, van Lier, Frijns, Meeus, \& Koot, 2011; Schwerdtfeger \& Friedrich-Mai, 2009; Thompson et al., 2012). These studies suggest that, in addition to displaying higher variability, depressed individuals experience more frequent affective fluctuations, or lower temporal dependency of affect.

However, research focusing on the temporal dependency of affect, labeled inertia, seems to contradict evidence of greater affective instability in depression; several studies have found depression to be associated with higher inertia, measured as the autocorrelation (ACORR) of affect over time (e.g., Koval, Kuppens, Allen, \& Sheeber, 2012; Kuppens, Allen, et al., 2010; Kuppens et al., 2012; Wenze, Gunthert, Forand, \& Laurenceau, 2009; but see Thompson et al., 2012). These findings suggest that depression is associated with greater predictability or resistance to affective change from moment to moment.

As illustrated by the studies reviewed above, the literature on affect dynamics in relation to depression presents a paradox: depression has been related to increased affective variability, instability and inertia. Yet, how can depressed individuals be at once affectively variable, labile, and inert? We propose that previous studies have not provided a conclusive test of the association between affect dynamics and depression because they have overlooked at least one of the following three important factors: a) dependencies between indices of affect dynamics; b) measurement of affect at different timescales; and c) the influence of external events.

\section{Dependencies Between Measures of Affect Dynamics}

Affective variability, instability, and temporal dependency have been investigated as distinct measures, yet they are not independent. The mathematical relationship between these three measures is captured by the following equation, derived by Jahng et al. (2008):

$$
\delta^{2}=2 \sigma^{2}(1-\rho(1))
$$

Here, the instability (i.e., MSSD, $\delta^{2}$ ) of a time series is expressed as a function of its variability (i.e., variance, $\sigma^{2}$ ) and temporal dependency [i.e., first-order ACORR, $\rho(1)$ ], meaning that "a high value of temporal instability. . is obtained when the series has high variability and low temporal dependency" (Jahng et al., 2008 , p. 356). However, each measure of affect dynamics is typically examined in isolation, making it difficult to assess how each uniquely relates to depression, and possibly giving rise to inconsistent findings across studies. This is particularly problematic for the MSSD, which may increase either as a function of higher variability, lower temporal dependency (inertia), or a combination of both (Wang et al., 2012). Thus, the association between depression and higher MSSD may, for instance, be fully accounted for by higher variability, and not by lower inertia (Thompson et al., 2012). This would imply that individuals with depression experience a larger range of affect levels, but not more frequent shifts in affect. On the other hand, depression may also be associated with both higher variability (e.g., Wichers et al., 2010) and higher inertia (e.g., Kuppens, Allen, et al., 2010), implying large but slow shifts in affect. It is crucial to note that because instability may be driven by increased variability alone (Wang et al., 2012), the combination of high variability and high inertia could also manifest itself in a relatively higher MSSD among depressed individuals. These dependencies between measures imply that examining each measure in isolation may obfuscate their true associations with depression. Thus, to determine whether depression is truly associated with higher affective instability — versus merely higher variability, or both higher variability and higher inertia-it is necessary to examine how the two components of instability (i.e., variability and inertia) uniquely relate to depression.

This could be achieved using multiple regression, where variability (i.e., $S D$ ) and inertia (i.e., ACORR) of affect were entered as simultaneous predictors of depression. However, given that the equation above can be rewritten to express each measure of affect dynamics as a function of the other two (e.g., variability can be expressed as a function of inertia and instability), it may also be informative to predict depression simultaneously from the other two pairs of dynamic measures. For instance, regressing depression simultaneously onto instability and variability would reveal whether the positive association between depression and instability is driven exclusively by higher variability. If this were the case, instability should no longer be associated with depression after controlling for variability. While such a multiple regression approach may not control for all the possible confounds arising from the mathematical dependencies between measures, it would provide a first important step toward revealing the unique associations between each component of affect dynamics and depression. This approach also makes it possible to control for mean level of affect, which is important when examining affect dynamics (EbnerPriemer et al., 2009).

\section{Timescale}

Another important factor relating to the study of affect dynamics is the timescale on which affective change is considered (Hollenstein et al., 2013). Previous studies have assessed affect at timescales ranging from seconds (Kuppens, Allen, et al., 2010) to days (Neumann et al., 2011). This methodological diversity may produce disparate findings for two reasons: First, a given process may appear very different when measured at various sampling frequencies (Ebner-Priemer \& Sawitzki, 2007). For instance, observing the sun's position in the sky once a day versus every hour would produce vastly different profiles of change. Second, measuring feelings at different timescales may actually capture different affective processes. For example, moods and emotions are often 
distinguished by their duration, with moods lasting longer and being slower to change than emotions (Rosenberg, 1998). Hence, affective changes over hours or days may be driven primarily by mood-related processes, whereas dynamics at shorter timescales may be more likely to be governed by emotion-related processes (Rottenberg, 2005). By implication, the association between depression and higher inertia at shorter timescales (e.g., Koval \& Kuppens, 2012; Kuppens, Allen, et al., 2010) may reflect insensitivity to the changing emotional context (Rottenberg, 2005), whereas higher instability among depressed individuals at longer timescales (e.g., Thompson et al., 2012) may reflect mood swings. Emotional inertia and mood instability could conceivably cooccur, perhaps even within the same individual. Thus, taking into account the timescale is crucial when interpreting associations between depression and affect dynamics.

\section{The Influence of Events}

Previous studies of affect dynamics in depression have predominantly used the experience sampling method (ESM) or similar naturalistic methods (e.g., Koval \& Kuppens, 2012; Wichers et al., 2010). While these methods offer many advantages, including high ecological validity, they do not afford proper control over events. This is problematic because observed differences in affect dynamics may result either from environmental differences (i.e., encountered events) or from differences in emotional responding and regulation (Koole, 2009). Indeed, depression is not only associated with altered reactivity to emotional events (e.g., Bylsma, TaylorClift, \& Rottenberg, 2011) and impairments in emotion regulation (Gross \& Muñoz, 1995), but also with the experience of more frequent stressful events (Hammen, 2005). Each of these may contribute to the alterations in affect dynamics observed in depression. Controlling for the influence of external events is an important step toward disentangling the role of context versus more endogenous factors. Thus, studies on individual differences in affect dynamics using tightly controlled laboratory designs, in which all participants encounter the same sequence of events, are urgently needed. Exposing all participants to a series of emotional stimuli in a fixed order would help rule out the influence of differential exposure to events in determining differences in affect dynamics. For instance, previous studies linking depressive symptoms with increased NA inertia in daily life (e.g., Koval et al., 2012) cannot rule out the possibility that higher inertia was driven by differences in the type or sequence of events encountered by more depressed individuals.

\section{The Current Study}

The current study used a novel paradigm and analytic approach to address the limitations discussed above. Specifically, we designed a task in which participants rated their affect in response to a series of emotional film clips presented in a fixed order in the lab. This paradigm measured feelings in response to a specific sequence of events on a timescale of minutes and, therefore, clearly captured the dynamics of emotions (vs. moods). Participants also reported their feelings in daily life 10 times a day for 1 week using ESM, providing a naturalistic comparison for our laboratory findings. Participants were preselected to represent a wide range of depressive symptoms based on a prescreening questionnaire. We used multiple regression to assess how depressive symptoms were uniquely associated with measures of variability, inertia, and instability derived from both the film-clip task and ESM.

Few studies have directly compared how depression relates to the dynamics of NA versus PA, with most either focusing exclusively on NA (e.g., Koval et al., 2012; Silk et al., 2003) or using bipolar scales (e.g., Cowdry, Gardner, O’Leary, Leibenluft, \& Rubinow, 1991; Golier, Yehuda, Schmeidler, \& Siever, 2001). However, some research suggests that depression may be more strongly related to alterations in NA dynamics (e.g., Thompson et al., 2012; Wichers et al., 2010), and this is supported by the findings of a recent meta-analysis (Houben, Kuppens, \& Van Den Noortgate, 2013). In line with these findings, we predicted that depressive symptoms would be more strongly related to NA dynamics. Also in line with previous research, we predicted that depressive symptoms would be positively related to all measures of NA dynamics when examined using simple correlations. However, in contrast to previous findings, we expected these associations to change when controlling for overlap between dynamic measures using multiple regression. Specifically, we predicted that depressive symptoms would be independently related to higher NA inertia (e.g., Kuppens, Allen, et al., 2010; Kuppens et al., 2012) and NA variability (e.g., Wichers et al., 2010). Due to the relationship between dynamic measures (see equation 1), this prediction also implies that the association between NA instability and depressive symptoms should become negative after controlling for variability. Put otherwise, statistically removing the variability component of instability leaves the inverse of inertia. If depressive symptoms are related to higher NA inertia, they should be negatively associated with NA instability once variability is partialed out. We did not make strong a priori hypotheses regarding differences in results from the film-clip task versus ESM. Rather, measuring participants' affect dynamics using both methods allowed us to explore how timescale and exposure to events influenced associations between affect dynamics and depressive symptoms.

\section{Method}

\section{Participants and Prescreening}

An initial pool of 439 undergraduates at the KU Leuven were screened on depressive symptoms using the Center for Epidemiologic Studies Depression Scale (CES-D; Radloff, 1977; Range = $0-52, M=16.39, S D=10.27, \alpha=.92$ ). We aimed to recruit 100 participants representing a wide, balanced, and uniform range of depressive symptoms. This sample size was selected to ensure sufficient power for detecting expected effect sizes (i.e., correlations around .3) and based on the budget available for paying participants. We adopted a stratified sampling approach (Ingram \& Siegle, 2009), whereby the CES-D prescreening range was divided into five roughly equal segments, and a random sample of participants from each segment were contacted. We contacted 241 eligible participants before reaching our target sample of 100 (CES-D prescreening Range $=0-50 ; M=19.27 ; S D=12.53$ ). The final sample included 55 participants scoring above Radloff's (1977) clinical cutoff score (CES-D $\geq 16$ ), and 32 participants who scored above the more conservative clinical cutoff $(C E S-D \geq 27)$ 
proposed by Gotlib, Lewinson, and Seeley (1995). ${ }^{2}$ Participants were recruited as part of a larger study investigating different aspects of emotional functioning for which they received $70 €$. One participant withdrew early, leaving a final sample of 99 (62 women; $\left.M_{\text {age }}=19.05, S D_{\text {age }}=1.27\right)$. ESM data from four additional participants were excluded due to equipment malfunction $(n=3)$ or poor compliance with the ESM protocol $(n=1)$. No other data exclusions were administered. ${ }^{3}$

\section{Materials and Procedure}

At an initial lab session, participants once again completed the CES-D to assess their current depressive symptoms, among other personality, well-being, cognitive control, and resting physiology measures. No other measures or manipulations were administered. Participants then reported their daily experiences of PA and NA 10 times/day for 7 days using ESM. At the end of the week, participants returned to the lab for a final session during which they completed the film-clip task.

Depressive symptoms. The CES-D was used to measure current depressive symptoms. This 20-item scale asks respondents to indicate how frequently they have experienced a range of depressive symptoms (e.g., "I had crying spells") over the past week from 0 (rarely or none of the time) to 3 (most or all of the time). Descriptives for the CES-D are displayed in Table 1. At the time of the study, 37 participants scored at or above Radloff's (1977) clinical cutoff (CES-D $\geq 16$ ) whereas only 13 participants scored at or above the more conservative clinical cutoff (CES-D $\geq 27)$ proposed by Gotlib et al. (1995). The scale showed very good reliability $(\alpha=.92)$ and correlated with CES-D at prescreening at $r(99)=.72, p<.001$. All analyses reported below used CES-D scores measured at the time of the study, not the CES-D scores measured at prescreening.

Film-clip task. The task was programmed using E-Prime (Psychology Software Tools, Inc.). Participants completed the task in individual cubicles with headphones, on PCs with 17-inch color CRT monitors $(1024 \times 768$ resolution; $60 \mathrm{~Hz}$ refresh rate). The task began with the following instructions:

During this task you will watch several film clips. Each film clip will be followed by a number of questions asking you how you are feeling at the moment. Please rate how you feel after watching each film clip, not how you are feeling today, or how you feel in general, or how you think people should feel after seeing each film clip. . . Please note that you will have a maximum of $7 \mathrm{~s}$ to complete each question.

These instructions aimed to reduce demand effects and ensure that participants reported emotions rather than moods or affective traits. Before the main task began, participants viewed a neutral film clip and were given the chance to practice completing ratings in the allotted time. Participants then completed baseline affect ratings, after which they viewed 10 film clips presented in a fixed sequence and rated their affect following each clip. Film clips were selected from a validated database of 70 emotion-eliciting film excerpts (Schaefer, Nils, Sanchez, \& Philippot, 2010) and comprised four positive, four negative, and two neutral clips, shown in the following fixed order: Trainspotting[1] (negative), Schindler's List[3] (negative), Blue[2] (neutral), Trainspotting[3] (positive), Blue[3] (neutral), The Dentist (negative), Benny and Joon (positive), There is Something About Mary[1] (positive), Indiana Jones and the Last Crusade (negative), When a Man Loves a Woman (positive). We selected these clips based on emotion ratings from Schaefer et al.'s (2010) validation study. To avoid ceiling effects, the selected film clips were not among the 20 highest on "emotional arousal" in Schaefer et al.'s (2010) validation study. To limit the overall length of the task, the selected clips were shorter than 3 min (Range $=0: 25-2: 28 \mathrm{~min}$ ). Finally, only English-speaking film clips were used and Dutch subtitles were added. Affect ratings were made on two items measuring PA (happy, relaxed) and four items measuring NA (sad, depressed, anxious, angry) rated on a scale from 0 (not at all) to 6 (very much).

ESM protocol. Participants received a Palm Tungsten E2 palmtop that was programmed using the Experience Sampling Program (Barrett \& Barrett, 2001) to beep 10 times/day for 7 days, according to a stratified random interval scheme. On average, participants were beeped every $73.30 \mathrm{~min}(S D=29.33)$. Compliance was high: participants responded to an average of $91.5 \%$ of the programmed beeps $(S D=6.2)$. At each beep, participants rated their current PA and NA using the same items as in the film-clip task, with responses on a continuous slider scale from 1 (not at all) to 100 (very much).

Affect dynamics. Separately for the film-clip and ESM data, we formed PA and NA scales by averaging scores on the two positive and four negative feeling items, respectively. We then calculated the following within-person measures of affect dynamics separately for PA and NA: the within-person $S D$ was used as a measure of affective variability (Wichers et al., 2010). Affective instability was calculated as the RMSSD (the square root of the MSSD), which expresses instability on the original scale of the affect items and also corrected for the positively skewed MSSD scores (Jahng et al., 2008). The ACORR was used to measure the temporal dependency or inertia of affect (Kuppens, Allen, et al., 2010). Finally, we calculated the mean level of PA and NA to include as an additional control variable in our analyses.

\section{Results}

\section{Film Clip Manipulation Check}

To check that the film clips successfully induced the intended emotional valence, we analyzed participants' PA and NA ratings using two separate repeated-measures ANOVAs with film-valence (positive vs. negative vs. neutral) as the within factor. Both models were highly significant: $F \mathrm{~s}>127, p s<$. 001. Follow-up comparisons indicated that positive films elicited significantly higher levels of PA than negative and neutral films, whereas negative films induced significantly greater NA than positive and neutral films $(p s<.001)$.

\section{Simple Correlation Analyses}

Pearson correlations were calculated between CES-D scores and all measures of affect dynamics from both the film-clip task and

\footnotetext{
${ }^{2}$ CES-D scores reported from the prescreening were used for recruitment purposes only and are not the scores used in the main analyses (see below).

${ }^{3}$ All analyses were conducted using the maximum available sample size: for analyses involving ESM data $n=95$; for all other analyses $n=99$.
} 


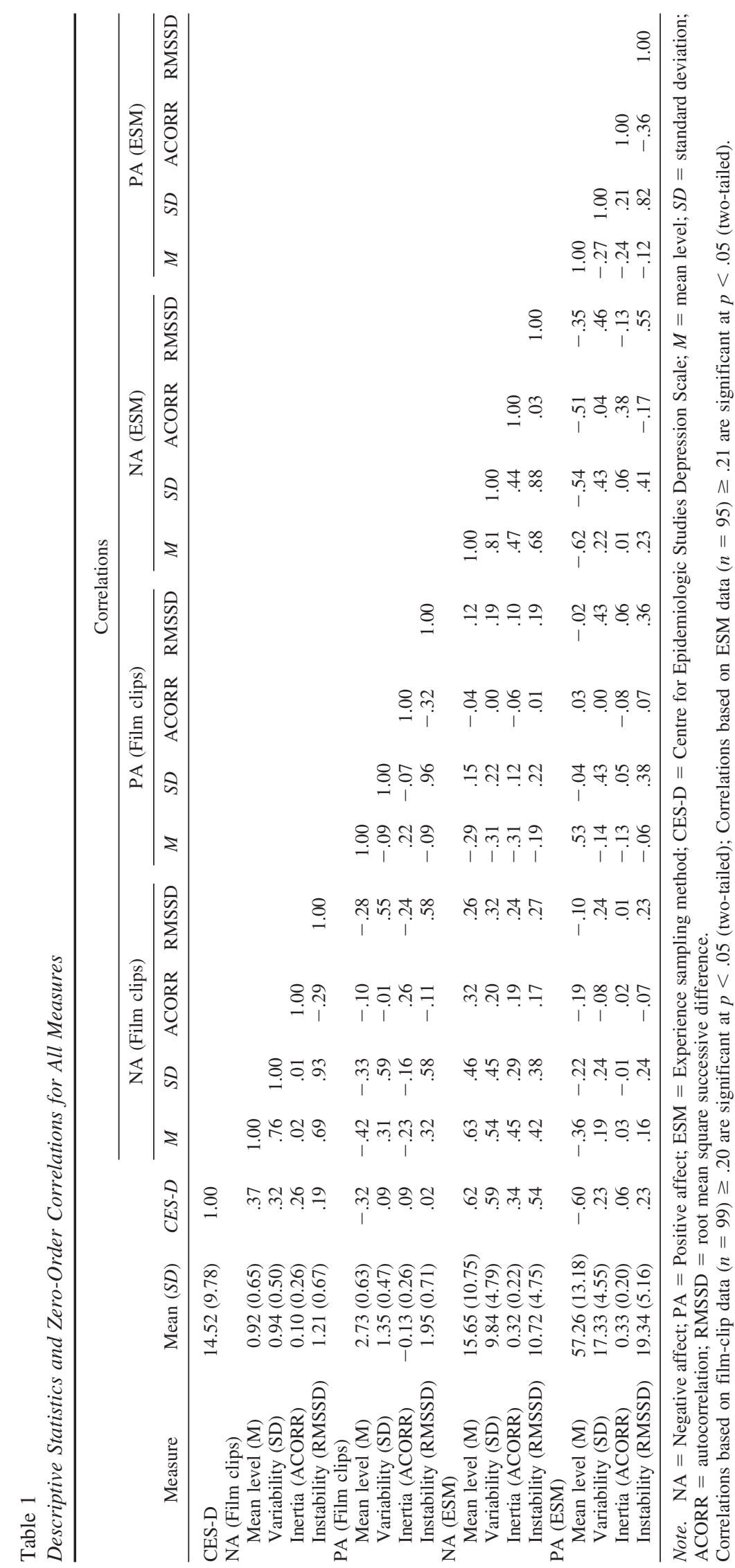


ESM. These correlations, representing effect sizes for bivariate associations between depressive symptoms and each measure of affect dynamics, are presented in Table 1 together with descriptive statistics for all study variables.

Film-clip task. Regarding film-clip dynamics, depressive symptoms were associated with higher inertia $(p=.01)$, variability $(p=.001)$ and instability $(p=.061)$ of NA, whereas none of the PA dynamic measures from the film-clip task correlated with the CES-D $(p s>.35)$. Depressive symptoms were also related to higher mean NA and lower mean PA $(p s<.01)$.

ESM. We found similar results for NA dynamics based on ESM data: depressive symptoms correlated positively with NA inertia $(p=.001)$, variability $(p<.001)$ and instability $(p<$ $.001)$. However, in contrast to the film clips, depressive symptoms were also positively associated with PA variability ( $p=$ $.03)$ and instability $(p=.02)$. Finally, depressive symptoms were related to higher mean NA and lower mean PA in daily life $(p s<.001)$.

Associations between film-clip and ESM measures. Given that participants completed both the film-clip task and ESM, we were also able to examine whether affect (dynamic) measures obtained in the lab correlated with those measured in daily life. Mean levels of affect showed the strongest positive correlation across paradigms $(p s<.001)$. However, we also found (marginally) significant positive correlations between all dynamic measures from the ESM and those from the film-clip task $(p s<.07)$, with the exception of PA inertia which was unrelated across the two paradigms $(p=.42)$.

\section{Multiple Regression Analyses}

To examine whether the simple correlations between affect dynamics and depressive symptoms (reported above) were accounted for by overlap between dynamic measures, we ran two-step multiple regression analyses. In particular, we aimed to determine how variability and inertia were independently related to depressive symptoms. Thus, our main analyses consisted of regressing CES-D scores simultaneously onto $S D$ and ACORR. We also conducted ancillary analyses predicting CES-D scores simultaneously from the other two pairwise combinations of dynamic measures (given the mathematical relationships between dynamic measures, including all three measures in a single model would have resulted in problematic multicollinearity). At a second step of each model, we additionally controlled for mean level of affect. Separate analyses were conducted using data from the film-clip task and ESM, and separate models were conducted for dynamics of NA and PA. Tables 2 and 3 display results of the six multiple regression models in which pairs of NA dynamics were entered as simultaneous predictors of depressive symptoms. In these tables, standardized betas are effect sizes representing the unique association between each measure of NA dynamics and depressive symptoms after controlling for the other NA dynamic measure in the model (Step 1), and after additionally controlling for mean level of NA (Step 2). Finally, $R^{2}$ values in Tables 2 and 3 are effect sizes representing the proportion of variance in depressive symptoms accounted for by the combined predictors in each model.

Film-clip task. In a first multiple regression analysis, NA inertia and variability were entered as simultaneous predictors of CES-D scores (see Table 2). Both inertia and variability were significantly positively associated with depressive symptoms. However, after adding mean level of NA to the model (at Step 2 ), the effect of NA inertia remained significant, whereas the effect of variability became nonsignificant. Thus, both NA variability and inertia were independently associated with higher depressive symptoms, but only the association with inertia remained after controlling for mean NA. We ran two additional models predicting depressive symptoms from the other two pairs of affect dynamics. In Model 2, CES-D scores were regressed onto NA variability and instability: depressive

Table 2

Results of Multiple Regression Models Predicting Depression Severity From Dynamics and Mean Level of Negative Affect in the Film-Clip Task

\begin{tabular}{|c|c|c|c|c|c|c|}
\hline & \multicolumn{3}{|c|}{ Step 1} & \multicolumn{3}{|c|}{ Step 2 (including Mean level) } \\
\hline & $B(S E)$ & $\beta$ & $p$ & $B(S E)$ & $\beta$ & $p$ \\
\hline \multicolumn{7}{|l|}{ Model 1} \\
\hline Variability (SD) & $6.25(1.83)$ & .32 & .001 & $2.10(2.75)$ & .11 & .447 \\
\hline Inertia (ACORR) & $9.51(3.50)$ & .25 & .008 & $9.35(3.45)$ & .25 & .008 \\
\hline Mean level & - & - & - & $4.17(2.09)$ & .28 & .049 \\
\hline Adjusted $R^{2}$ & .150 & & $<.001$ & .175 & & $<.001$ \\
\hline \multicolumn{7}{|l|}{ Model 2} \\
\hline Variability (SD) & $22.18(5.02)$ & 1.13 & $<.001$ & $17.79(5.46)$ & .90 & .002 \\
\hline Instability (RMSSD) & $-12.55(3.71)$ & -.86 & .001 & $-12.15(3.66)$ & -.84 & .001 \\
\hline Mean level & - & - & - & $3.92(2.06)$ & .26 & .060 \\
\hline Adjusted $R^{2}$ & .182 & & $<.001$ & .204 & & $<.001$ \\
\hline \multicolumn{7}{|l|}{ Model 3} \\
\hline Inertia (ACORR) & $12.74(3.71)$ & .34 & .001 & $9.62(3.81)$ & .26 & .013 \\
\hline Instability (RMSSD) & $4.16(1.43)$ & .29 & .005 & $0.37(2.04)$ & .03 & .855 \\
\hline Mean level & - & - & - & $5.11(2.01)$ & .34 & .013 \\
\hline Adjusted $R^{2}$ & .123 & & .001 & .170 & & $<.001$ \\
\hline
\end{tabular}

Note. $S D=$ standard deviation; ACORR $=$ autocorrelation; RMSSD $=$ root mean square successive difference.

$N=99$ for all regression models. 
Table 3

Results of Multiple Regression Models Predicting Depression Severity From Dynamics and Mean Level of Negative Affect in the ESM

\begin{tabular}{|c|c|c|c|c|c|c|}
\hline & \multicolumn{3}{|c|}{ Step 1} & \multicolumn{3}{|c|}{ Step 2 (including Mean level) } \\
\hline & $B(S E)$ & $\beta$ & $p$ & $B(S E)$ & $\beta$ & $p$ \\
\hline \multicolumn{7}{|l|}{ Model 4} \\
\hline Variability (SD) & $1.11(0.19)$ & .55 & $<.001$ & $0.53(0.28)$ & .26 & .058 \\
\hline Inertia (ACORR) & $4.39(4.02)$ & .10 & .277 & $1.93(3.99)$ & .04 & .629 \\
\hline Mean level & - & - & - & $0.35(0.13)$ & .38 & .007 \\
\hline Adjusted $R^{2}$ & .347 & & $<.001$ & .390 & & $<.001$ \\
\hline \multicolumn{7}{|l|}{ Model 5} \\
\hline Variability (SD) & $1.10(0.36)$ & .54 & .003 & $0.32(0.43)$ & .16 & .451 \\
\hline Instability (RMSSD) & $0.11(0.36)$ & .06 & .752 & $0.23(0.35)$ & .12 & .502 \\
\hline Mean level & - & - & - & $0.37(0.12)$ & .41 & .004 \\
\hline Adjusted $R^{2}$ & .339 & & $<.001$ & .391 & & $<.001$ \\
\hline \multicolumn{7}{|l|}{ Model 6} \\
\hline Inertia (ACORR) & $14.04(3.53)$ & .32 & $<.001$ & $7.68(4.36)$ & .18 & .081 \\
\hline Instability (RMSSD) & $1.07(0.17)$ & .52 & $<.001$ & $0.63(0.25)$ & .31 & .012 \\
\hline Mean level & - & - & - & $0.29(0.12)$ & .33 & .019 \\
\hline Adjusted $R^{2}$ & .378 & & $<.001$ & .408 & & $<.001$ \\
\hline
\end{tabular}

Note. $S D=$ standard deviation; ACORR $=$ autocorrelation; RMSSD $=$ root mean square successive difference.

$N=95$ for all regression models.

symptoms were significantly positively associated with NA variability, whereas the association with NA instability was significantly negative (see Table 2). ${ }^{4}$ Results did not change after controlling for mean NA at Step 2. Recall that the simple correlation between depressive symptoms and NA instability was positive (see Table 1). Thus, after partialing out the variability component of instability, the remaining component was negatively associated with depressive symptoms. This is consistent with the results of Model 1, in which both variability and inertia were independently positively related to depressive symptoms. In Model 3, NA inertia and instability were entered together as simultaneous predictors; both inertia and instability were independently positively associated with depressive symptoms (see Table 2). After controlling for NA mean level at Step 2 , the effect of inertia remained significant, whereas instability was no longer related to depressive symptoms. Mean level of NA independently predicted depressive symptoms in all three models. We did not run multiple regressions for film-clip PA dynamics as none of the PA dynamic measures correlated with depressive symptoms (see Table 1).

ESM. As for the film-clip data, we first regressed CES-D scores onto NA inertia and variability. Depressive symptoms were independently associated with significantly higher NA variability. However, the association between depressive symptoms and NA inertia was not significant (see Model 4 in Table 3). After mean level of NA was added to the model at Step 2, the association with NA variability became marginally significant. We again ran two additional models predicting depressive symptoms from the other two pairs of affect dynamics. In Model 5, CES-D scores were positively associated with NA variability, but were unrelated to instability of NA (see Table 3). However, even the effect of variability became nonsignificant after controlling for NA mean level at Step 2. In Model 6, both inertia and instability showed independent positive associations with depressive symptoms (see Table 3). After controlling for NA mean level (Step 2), inertia remained a marginally significant predictor of higher depressive symptoms, and instability remained significantly positively related to depressive symptoms. Again, NA mean level was positively related to depressive symptoms in all three Models, independent of NA dynamics. Finally, because variability and instability of PA in the ESM were correlated with depressive symptoms (see Table 1), we conducted a final regression model predicting CES-D scores simultaneously from PA variability and instability. Neither PA instability nor variability showed independent associations with depressive symptoms $(p s>.42)$.

Analyses using dichotomized CES-D scores. The above findings are not directly analogous to the findings of previous studies that have compared affect dynamics among clinically depressed versus healthy participants (e.g., Thompson et al., 2012). However, to approximate such a comparison we conducted additional analyses using dichotomized CES-D scores. ${ }^{5}$ Specifically, we compared participants reporting potentially clinically significant levels of depressive symptoms (i.e., CES-D $\geq 27, n=13$; see Gotlib et al., 1995) versus those with low levels of depressive symptoms (CES-D $\leq 9, n=37$ ). However, because so few participants scored at or above Gotlib et al.'s (1995) proposed CES-D clinical cutoff, we also repeated the analyses using Radloff's (1977) lower clinical cutoff (CES-D $\geq 16, n=37$ ). Using logistic regression, we repeated Models 1-6 (see above) with high versus low CES-D group (coded as $1=$ high CES-D; $0=$ low CES-D) as the dichotomous outcome. All effects at Step 1 of Models 1-6 remained in the same direction and statistically (non)significant when using dichotomized CES-D scores as the outcome. At Step 2 of each model, all effects were in the same direction as in the original analyses. However, some previously significant effects became marginally significant or vice versa. In sum, the analyses

\footnotetext{
${ }^{4}$ Although $S D$ and RMSSD were highly correlated (see Table 1), VIFs were $<8$ (see Myers, 1990). Furthermore, repeating Model 2 with random subsamples of $60 \%, 70 \%, 80 \%$, and $90 \%$ of participants did not change results, suggesting that multicollinearity was not problematic.

${ }^{5}$ We thank an anonymous reviewer for suggesting these additional analyses using dichotomized CES-D scores.
} 
using dichotomized CES-D scores (with either 27 or 16 as a cutoff for the high CES-D group) closely replicated our main findings.

\section{Discussion}

In the current study, we examined how depressive symptoms are related to affective changes in response to a fixed sequence of events on a timescale of minutes (film-clip task) versus naturalistic affective fluctuations in daily life on a timescale of hours (ESM), while statistically controlling for overlap between different dynamic measures. This approach aimed to pinpoint the specific patterns of affect dynamics associated with depressive symptoms and thus suggest a way to reconcile previous inconsistencies in the literature.

In terms of simple correlations, our results replicated the paradoxical results of previous studies: depressive symptoms were positively related to variability, inertia, and instability of NA both in the lab (film-clip task) and in daily life (ESM). These findings also line up with a recent meta-analysis of the literature on affect dynamics and well-being, in which depression was found to be associated with significantly greater variability, instability, and inertia of NA (Houben et al., 2013). However, after controlling for dependencies between measures of affect dynamics using multiple regression, a different pattern of results emerged.

Multiple regression analyses showed that depressive symptoms were independently related to both higher inertia and higher variability of NA in response to the film clips (see Model 1). Yet, only the association with inertia remained significant after controlling for mean NA. These findings suggest that, at a short timescale and controlling for external events, depressive symptoms were related to the tendency to experience more intense negative feelings that were more resistant to change over time. Due to the mathematical relationships between dynamic measures, these findings imply that the association between depressive symptoms and NA instability should become negative after controlling for the effect of NA variability, which is exactly what we found in Model 2. This reversal of the relationship between instability and depressive symptoms is due to the fact that partialing out the variability component of instability leaves the inverse of temporal dependency (i.e., low inertia—see Equation 1); since we found depressive symptoms to be related to higher inertia, it follows that the inverse of inertia correlates negatively with depressive symptoms.

Similar analyses of the ESM data revealed that only NA variability was independently related to depressive symptoms, whereas neither inertia nor instability showed unique associations with CES-D scores after controlling for variability (see Models 4 and 5 ). Thus, in terms of affective fluctuations in daily life on a scale of hours, and not necessarily tied to specific events, depressive symptoms appear to be exclusively related to a larger overall range of negative feelings. However, we note this effect was partly accounted for by higher mean level of NA (see Step 2 of Models 4 and 5). Importantly, this finding does not imply that depressive symptoms are associated with higher affective instability, defined as the combination of higher variability and lower temporal dependency (Jahng et al., 2008; Wang et al., 2012). Rather, the current ESM findings line up with Thompson et al.'s (2012) results, in which participants with MDD had higher variability but comparable inertia of NA in daily life, relative to healthy controls. The results of Model 6 are less straightforward to interpret: both
NA inertia and instability (in the ESM) showed independent positive associations with depressive symptoms, yet neither had a significant independent effect (over and above variability) in Models 4 and 5. We speculate that this may be driven by the relationship between variability and depressive symptoms; RMSSD and ACORR may become significant predictors when entered together because of their overlap with $S D$ (see Table 1).

Taken together, the current findings provide partial support for our hypotheses. As predicted, depressive symptoms were independently associated with both higher variability and inertia of NA. However, the association with inertia was only robust for shortterm emotional changes in response to a fixed sequence of events, as captured by the film-clip task. These findings line up with previous studies linking depression with higher variability (e.g., Wichers et al., 2010) and inertia (e.g., Kuppens, Allen, et al., 2010). Importantly, these findings also clarify how depression can be related to higher inertia, higher variability, and higher instability of affect at the same time. While it is possible to imagine a person whose affect is both variable and inert (i.e., large but slow changes in affect), it is more difficult to see how affective inertia and instability could coexist. However, because of the strong dependency between instability (as measured by the MSSD) and variability (as measured by the $S D$ ), having higher variability may be sufficient to inflate a person's level of instability, regardless of their level of inertia.

The current findings have a number of implications for the study of affect dynamics. First and foremost, our findings highlight the importance of distinguishing between affect dynamics at different timescales. While this issue has received relatively little attention in the literature, it is essential for understanding individual differences in affect dynamics (Hollenstein et al., 2013; Rottenberg, 2005). For instance, we found depressive symptoms to be independently related to higher NA autocorrelation in the film-clip task, but not in the ESM. This implies that depressive symptoms are associated with higher inertia of negative emotions but not necessarily of negative moods. In contrast, increased NA variability was related to depressive symptoms in both the film-clip task and ESM, suggesting that it may apply to both emotions and moods.

In a related vein, the current study's findings suggest that the role of contextual factors, such as differential exposure to events, should be given greater consideration in research on affect dynamics. Specifically, as suggested by differences in results from the film-clip task and ESM, the events to which people are exposed may play a large role in determining how their feelings fluctuate across time. This implies, for instance, that the association between depressive symptoms and higher NA inertia observed in the filmclip task must be due to endogenous processes (see Kuppens et al., 2012). As the current study cannot speak to which endogenous processes drive higher inertia of negative emotions, an important direction for future research will be to explore the mechanisms underlying emotional inertia.

The current study also indicates that accounting for the mathematical relationships between different indices of affect dynamics is crucial. As the results of the multiple regression analyses revealed, controlling for dependencies between affect dynamics may have a direct bearing on how they relate to measures of well-being, such as depressive symptoms. Similarly, our results underline the utility of controlling for differences in mean levels of affect when 
examining affect dynamics. The association between short-term NA inertia and depressive symptoms was independent of NA mean level. Thus, the inflexible negative emotional responding observed among more dysphoric participants is not merely due to their tendency to experience more intense negative emotions (Kashdan \& Rottenberg, 2010).

Although direct application of the current study's findings to clinical depression may be unwarranted given the lack of clinical diagnoses, our findings may have secondary implications for the conceptualization and treatment of MDD. For instance, therapies that aim to attenuate the intensity of a person's affective reactions may be beneficial in reducing affective instability, which is thought to result from hypersensitivity to certain environmental cues (Thompson, Berenbaum, \& Bredemeier, 2011). However, such treatments may be less effective for treating depression if it is characterized by emotional inertia, which may result in part from blunted emotional reactivity (see Bylsma, Morris, \& Rottenberg, 2008). In contrast, treatments that facilitate more rapid recovery from negative affective experiences may reduce emotional inertia. These examples are not exhaustive, but rather serve to illustrate that a more accurate and nuanced understanding of how affect dynamics are related to depression could help inform its treatment (Holtzheimer \& Mayberg, 2011).

The current study is not without limitations. First, the film-clip task measured emotional responses to standardized stimuli viewed passively in the lab, leaving open the possibility that our findings may not generalize to personally relevant emotional events. Indeed, this may have contributed to divergence between our results from the film-clip task and ESM. However, we note that most measures of affect dynamics based on the film-clip task correlated moderately with corresponding measures from the ESM, underscoring the validity of our novel lab assessment of affect dynamics. A second limitation relates to our reliance on self-report measures of emotions, which may be biased by demand characteristics. Despite all their shortcomings, self-reports are currently the only valid way of measuring subjective affective experiences (Barrett, Mesquita, Ochsner, \& Gross, 2007). In addition, while demand characteristics may influence self-reported emotions in response to particular stimuli (particularly in the lab), they are less likely to influence dynamical patterns in self-reported feelings over time. Finally, our sample was not characterized with respect to MDD. However, a number of factors mitigate this concern. First, our use of a stratified sampling approach ensured that a wide range of depressive symptom levels were represented, which increases the generalizability of our findings to clinical depression (Ingram \& Siegle, 2009). Second, depression is more likely to be dimensional than categorical (Haslam, Holland, \& Kuppens, 2012), suggesting that there is value in examining associations between affect dynamics and depressive symptom severity. Third, subthreshold depressive symptoms measured using the CES-D prospectively predict the onset of MDD, even after controlling for other known demographic, clinical, and psychosocial predictors (Klein et al., 2013). Furthermore, we replicated our analyses using dichotomized CES-D scores and found highly similar results. Nevertheless, it will be important to replicate our findings in a sample including participants with MDD.

Despite these limitations, the current study makes a number of important contributions. First, we introduce a novel paradigm for assessing affect dynamics in the lab. Although film clips are a standard tool for eliciting emotions in the laboratory (Rottenberg, Ray, \& Gross, 2007), we are not aware of previous studies that have examined the sequential effects of a series of film clips on emotions. In fact, when participants are shown multiple film clips, researchers are generally at pains to reduce spillover from one film to the next. In contrast, this study highlights the value in measuring emotional spillover/change across a series of film clips; it allows for the measurement of affect dynamics on shorter timescale than is possible using ESM, and ensures that all participants are exposed to the same sequence of emotional events. Furthermore, by measuring affect dynamics using the film-clip task and the ESM, the current study addressed an important gap in the literature regarding the relationship between emotional processes in the lab versus in daily life (Bylsma \& Rottenberg, 2011). Finally, the current study draws attention to three important, yet neglected, factors that should be examined in research on affect dynamics: dependencies between indices of affect dynamics, differences in the timescale on which affect is measured, and the influence of external events on affective fluctuations. In so doing, the current study forms a first step to reconciling the paradoxical findings of previous studies on the relationship between affect dynamics and depression.

\section{References}

American Psychiatric Association. (2000). Diagnostic and statistical manual of mental disorders (4th ed, text revision). Washington, DC: Author.

Barrett, L. F., \& Barrett, D. J. (2001). An introduction to computerized experience sampling in psychology. Social Science Computer Review, 19, 175-185. doi:10.1177/089443930101900204

Barrett, L. F., Mesquita, B., Ochsner, K. N., \& Gross, J. J. (2007). The experience of emotion. Annual Review of Psychology, 58, 373-403.

Bylsma, L. M., Morris, B. H., \& Rottenberg, J. (2008). A meta-analysis of emotional reactivity in major depressive disorder. Clinical Psychology Review, 28, 676-691. doi:10.1016/j.cpr.2007.10.001

Bylsma, L. M., \& Rottenberg, J. (2011). Uncovering the dynamics of emotion regulation and dysfunction in daily life with ecological momentary assessment. In I. Nyklicek, M. Zeelenberg, \& A. Vingerhoets (Eds.), Emotion regulation and well-being (pp. 225-244). New York, NY: Springer.

Bylsma, L. M., Taylor-Clift, A., \& Rottenberg, J. (2011). Emotional reactivity to daily events in major and minor depression. Journal of Abnormal Psychology, 120, 155-167. doi:10.1037/a0021662

Campbell-Sills, L., \& Barlow, D. H. (2007). Incorporating emotion regulation into conceptualizations and treatments of anxiety and mood disorders. In J. J. Gross (Ed.) Handbook of emotion regulation (pp. 542559). New York, NY: Guilford Press.

Cowdry, R. W., Gardner, D. L., O'Leary, K. M., Leibenluft, E., \& Rubinow, D. R. (1991). Mood variability: A study of four groups. American Journal of Psychiatry, 148, 1505-1511.

Ebner-Priemer, U. W., Eid, M., Kleindienst, N., Stabenow, S., \& Trull, T. (2009). Analytic strategies for understanding affective (in)stability and other dynamic processes in psychopathology. Journal of Abnormal Psychology, 118, 195-202. doi:10.1037/a0014868

Ebner-Priemer, U. W., \& Sawitzki, G. (2007). Ambulatory assessment of affective instability in borderline personality disorder: The effect of sampling frequency. European Journal of Psychological Assessment, 23, 238-247. doi:10.1027/1015-5759.23.4.238

Golier, J. A., Yehuda, R., Schmeidler, J., \& Siever, L. J. (2001). Variability and severity of depression and anxiety in post-traumatic stress disorder and major depressive disorder. Depression and Anxiety, 13, 97-100. doi:10.1002/da.1022 
Gotlib, I. H., Lewinsohn, P. M., \& Seeley, J. R. (1995). Symptoms versus a diagnosis of depression: Differences in psychosocial functioning. Journal of Consulting and Clinical Psychology, 63, 90-100. doi: 10.1037/0022-006X.63.1.90

Gross, J. J., \& Muñoz, R. F. (1995). Emotion regulation and mental health. Clinical Psychology: Science and Practice, 2, 151-164. doi: 10.1111/j.1468-2850.1995.tb00036.x

Hammen, C. (2005). Stress and depression. Annual Review of Clinical Psychology, 1, 293-319. doi:10.1146/annurev.clinpsy.1.102803.143938

Haslam, N., Holland, E., \& Kuppens, P. (2012). Categories versus dimensions in personality and psychopathology: A quantitative review of taxometric research. Psychological Medicine, 42, 903-920. doi:10.1017/ S0033291711001966

Hollenstein, T., Lichtwarck-Aschoff, A., \& Potworowski, G. (2013). A model of socioemotional flexibility at three time scales. Emotion Review. Advance online publication. doi:10.1177/1754073913484181

Holtzheimer, P. E., \& Mayberg, H. S. (2011). Stuck in a rut: Rethinking depression and its treatment. Trends in Neurosciences, 34, 1-9. doi: 10.1016/j.tins.2010.10.004

Houben, M., Kuppens, P., \& Van Den Noortgate. (2013). Emotion dynamics and well-being: A meta-analysis. Manuscript submitted for publication.

Ingram, R. E., \& Siegle, G. J. (2009). Methodological issues in the study of depression. In I. H. Gotlib \& C. L. Hammen (Eds.), Handbook of depression (pp. 69-92). New York, NY: Guilford Press.

Jahng, S., Wood, P. K., \& Trull, T. J. (2008). Analysis of affective instability in EMA: Indices using successive difference and group comparison via multilevel modeling. Psychological Methods, 13, 354-375. doi: $10.1037 / \mathrm{a} 0014173$

Kashdan, T. B., \& Rottenberg, J. (2010). Psychological flexibility as a fundamental aspect of health. Clinical Psychology Review, 30, 865-878. doi:10.1016/j.cpr.2010.03.001

Keltner, D., \& Kring, A. M. (1998). Emotion, social function, and psychopathology. Review of General Psychology, 2, 320-342. doi:10.1037/ 1089-2680.2.3.320

Klein, D. N., Glenn, C. R., Kosty, D. B., Seeley, J. R., Rohde, P., \& Lewinsohn, P. M. (2013). Predictors of first lifetime onset of major depressive disorder in young adulthood. Journal of Abnormal Psychology, 122, 1-6. doi:10.1037/a0029567

Koole, S. L. (2009). The psychology of emotion regulation: An integrative review. Cognition \& Emotion, 23, 4-41. doi:10.1080/ 02699930802619031

Koval, P., \& Kuppens, P. (2012). Changing emotion dynamics: Individual differences in the effect of anticipatory social stress on emotional inertia. Emotion, 12, 256-267. doi:10.1037/a0024756

Koval, P., Kuppens, P., Allen, N. B., \& Sheeber, L. (2012). Getting stuck in depression: The roles of rumination and emotional inertia. Cognition \& Emotion, 26, 1412-1427. doi:10.1080/02699931.2012.667392

Kuppens, P., Allen, N. B., \& Sheeber, L. (2010). Emotional inertia and psychological maladjustment. Psychological Science, 21, 984-991. doi: $10.1177 / 0956797610372634$

Kuppens, P., Oravecz, Z., \& Tuerlinckx, F. (2010). Feelings change: Accounting for individual differences in the temporal dynamics of affect. Journal of Personality and Social Psychology, 99, 1042-1060. doi:10.1037/a0020962

Kuppens, P., Sheeber, L. B., Yap, M. B., Whittle, S., Simmons, J. G., \& Allen, N. B. (2012). Emotional inertia prospectively predicts the onset of depressive disorder in adolescence. Emotion, 12, 283-289. doi:10.1037/ a0025046

Larsen, R. J. (1987). The stability of mood variability: A spectral analytic approach to daily mood assessments. Journal of Personality and Social Psychology, 52, 1195-1204. doi:10.1037/0022-3514.52.6.1195

Myers, R. (1990). Classical and modern regression with applications (2nd ed.). Boston, MA: Duxbury.
Neumann, A., van Lier, P. A. C., Frijns, T., Meeus, W., \& Koot, H. M. (2011). Emotional dynamics in the development of early adolescent psychopathology: A one-year longitudinal study. Journal of Abnormal Child Psychology, 39, 657-669. doi:10.1007/s10802-011-9509-3

Peeters, F., Berkhof, J., Delespaul, P., Rottenberg, J., \& Nicolson, N. A. (2006). Diurnal mood variation in major depressive disorder. Emotion, 6, 383-391. doi:10.1037/1528-3542.6.3.383

Radloff, L. S. (1977). The CES-D scale: A self-report depression scale for research in the general population. Applied Psychological Measurement, 1, 385-401. doi:10.1177/014662167700100306

Rosenberg, E. L. (1998). Levels of analysis and the organization of affect. Review of General Psychology, 2, 247-270. doi:10.1037/ 1089-2680.2.3.247

Rottenberg, J. (2005). Mood and emotion in major depression. Current Directions in Psychological Science, 14, 167-170. doi:10.1111/j.09637214.2005.00354.x

Rottenberg, J., \& Johnson, S. L. (Eds.). (2007). Emotion and psychopathology: Bridging affective and clinical science. Washington, DC: American Psychological Association. doi:10.1037/11562-000

Rottenberg, J., Ray, R. D., \& Gross, J. J. (2007). Emotion elicitation using films. In J. A. Coan \& J. J. B. Allen (Eds.), The handbook of emotion elicitation and assessment (pp. 9-28). London, UK: Oxford University Press.

Schaefer, A., Nils, F., Sanchez, X., \& Philippot, P. (2010). Assessing the effectiveness of a large database of emotion-eliciting films: A new tool for emotion researchers. Cognition \& Emotion, 24, 1153-1172. doi: 10.1080/02699930903274322

Schwerdtfeger, A., \& Friedrich-Mai, P. (2009). Social interaction moderates the relationship between depressive mood and heart rate variability: Evidence from an ambulatory monitoring study. Health Psychology, 28, 501-509. doi:10.1037/a0014664

Silk, J. S., Steinberg, L., \& Morris, A. M. (2003). Adolescents' emotion regulation in daily life: Links to depressive symptoms and problem behavior. Child Development, 74, 1869-1880. doi:10.1046/j.1467-8624 .2003.00643.x

Thompson, R. J., Berenbaum, H., \& Bredemeier, K. (2011). Crosssectional and longitudinal relations between affective instability and depression. Journal of Affective Disorders, 130, 53-59. doi:10.1016/j .jad.2010.09.021

Thompson, R. J., Mata, J., Jaeggi, S. M., Buschkuehl, M., Jonides, J., \& Gotlib, I. H. (2012). The everyday emotional experience of adults with major depressive disorder: Examining emotional instability, inertia, and reactivity. Journal of Abnormal Psychology, 121, 819-829.doi:10.1037/a0027978

Wang, L. P., Hamaker, E., \& Bergeman, C. S. (2012). Investigating inter-individual differences in short-term intra-individual variability. Psychological Methods, 17, 567-581. doi:10.1037/a0029317

Watson, D., Clark, L. A., \& Carey, G. (1988). Positive and aegative affectivity and their relation to anxiety and depressive disorders. Journal of Abnormal Psychology, 97, 346-353. doi:10.1037/0021843X.97.3.346

Wenze, S. J., Gunthert, K. J., Forand, N. R., \& Laurenceau, J.-P. (2009). The influence of dysphoria on reactivity to naturalistic fluctuations in anger. Journal of Personality, 77, 795-824. doi:10.1111/j.1467-6494 2009.00565.

Wichers, M., Peeters, F., Geschwind, N., Jacobs, N., Simons, C. J. P., Derom, C., . . . van Os, J. (2010). Unveiling patterns of affective responses in daily life may improve outcome prediction in depression: A momentary assessment study. Journal of Affective Disorders, 124, 191195. doi:10.1016/j.jad.2009.11.010

Received October 25, 2012

Revision received May 24, 2013

Accepted May 29, 2013 